\title{
Dispositif De Formation Et D’évaluation Du Stage De Fin De Cycle : Premiers Constats
}

\author{
Somaya EI Gharras, MA \\ Jamila Charki, $M A$ \\ Université Cadi Ayyad, École Normale Supérieure de Marrakech, Maroc \\ doi: 10.19044/esj.2016.v12n1p335 URL:http://dx.doi.org/10.19044/esj.2016.v12n1p335
}

\begin{abstract}
The evolution of society brings new requirements for the citizen of tomorrow and consequently to the future's school. It is the duty of every society to worry about the support provided for the citizen's construction. The school being a key in the development of the individual, the main actors of this system must be properly supported in their training. The reform concerning training of teachers in Morocco is a part of the professionalization process through the creation of Regional Centers of Educational Professions and Training (CRMEF) who have a qualifying mission. These centers complement the training begun in the university education tracks (FUE). Previous training of Moroccan teacher who was held in the teacher training schools (ENS) included a significant practical training. However, capitalization of experiences from one year to another was insufficient. Leaving few traces the efforts made were not shown at fair value. In this work, we stopped on training, coaching and evaluation methods used on the Master specialized on educational professions provided in teacher training school (ENS) which is an institution in Cadi Ayyad University at Marrakesh. In this context, we propose a system which takes into account the different components of the activity of training. We assume that one of the advantages of this device is the reflexive dimension brought by the competence approach both for the teacher and the student. A second interest lies in the method adopted to evaluate the productions of trainees, like a competency referential to validate through a distance learning platform.
\end{abstract}

Keywords: Skills, professionalization, evaluation, training, teacher

Résumé

$\mathrm{Au}$ Maroc, la démarche de professionnalisation du métier d'enseignant est enclenchée par l'adoption de l'approche par compétences 
dans le système éducatif et, par conséquent, dans la formation de l'enseignant qui se concrétise dans les Centres Régionaux des Métiers de l'Éducation et de la Formation (CRMEF) par une qualification professionnelle. Ces centres complètent la formation entamée dans le cadre des filières universitaires d'éducation (FUE) où une place importante est donnée aux sciences de l'éducation, à la didactique des disciplines, ainsi qu'à un stage pratique dans le domaine de l'enseignement. Jusqu'en 2010, la formation de l'enseignant marocain du cycle secondaire qualifiant se déroulait en fin de parcours au sein des écoles normales supérieures (ENS). Toutefois, la capitalisation des expériences liées à la formation pratique était insuffisante. Ne laissant que peu de traces, les efforts engagés n'apparaissaient pas à leur juste valeur. Dans ce travail, nous nous sommes arrêtées sur les modalités de formation, d'accompagnement et d'évaluation dans le cadre du stage au niveau du Master d'Enseignement de l'École Normale Supérieure de Marrakech. Dans cette optique, nous avons mis en place un dispositif composé d'un référentiel de compétences, d'un système de formation et d'évaluation et d'une plateforme d'échange. La dynamique de ce dispositif a mis en exergue l'apport de l'évaluation formative adoptée. Un autre résultat important de ce dispositif réside dans la dimension réflexive qu'apporte la démarche adoptée aussi bien chez l'enseignant que chez l'étudiant.

Mots-clés: Compétences, professionnalisation, évaluation, formation, enseignant

\section{Introduction}

Nous tenons à préciser que, dans ce premier travail, nous ne nous situons pas dans une optique de comparaison entre les expériences antérieures et actuelle de formation et de suivi du stage de fin de cycle des filières universitaires d'éducation au Maroc. Nous nous proposons plus de tirer profit de l'expérience antérieure pour construire une méthodologie de travail qui intègre l'approche par compétences et le concept de professionnalisation du métier d'enseignant. Cet article n'a pas pour prétention d'évaluer le dispositif élaboré mais plutôt de rendre compte de l'expérience menée.

Dans la première partie, nous situons notre problématique dans le contexte national et international. Ensuite nous présentons des éléments théoriques soutenant nos propos et le cadre méthodologique adopté.

Dans la deuxième partie, nous donnons un descriptif du dispositif élaboré et de sa mise en œuvre. Enfin, nous citons les principaux retours de l'expérience exprimés par les stagiaires. 


\section{Éléments de fondement du dispositif de formation Contexte}

L'évolution de la société apporte de nouvelles exigences relatives au citoyen de demain et par voie de conséquence à l'école du futur. Il est du devoir de toute société de s'interroger sur l'accompagnement offert pour la construction de ce citoyen. L'école étant un environnement primordial dans le développement de l'enfant, les principaux acteurs de ce système doivent être correctement pris en charge dans leur formation.

$\mathrm{Au}$ Maroc, une étape essentielle dans la préparation au métier d'enseignant se déroule depuis peu au sein de l'université en l'occurrence via les filières universitaires d'éducation (FUE), cycle de licence et de Master.

Le Cahier des Normes Pédagogiques Nationales ${ }^{117}$ qui régit ces filières impose un stage en fin de formation. Celui-ci se doit d'intégrer un aspect pratique en rapport avec la spécialité, en l'occurrence l'enseignement et doit être accompagné d'une production écrite décrivant et analysant l'apport de cette activité. Dans le souci de développer la dimension opérationnelle du métier d'enseignant, une réflexion s'est engagée pour définir les modalités de mise en œuvre de cette exigence.

Ce travail s'inscrit dans une volonté d'innovation dans la formation initiale des enseignants et du développement de la recherche en relation avec l'ingénierie de la formation au Maroc.

\section{Compétences: vers une professionnalisation et une forme de professionnalisme de l'enseignant}

Dans le passé, l'exercice du métier d'enseignant relevait davantage de la notion de vocation et de gestes innés. Dans cet esprit, la formation de l'enseignant se résumait à un contenu scientifique et disciplinaire avec un accompagnement succinct en pédagogie à travers des enseignements théoriques en psychopédagogie et une mise en œuvre pratique du métier par le biais du stage en milieu professionnel.

La tendance actuelle va plutôt vers la professionnalisation du métier, dans le sens où, par des exercices théoriques et pratiques, le futur enseignant est amené à construire des compétences liées à sa profession. Cette tendance tient ses fondements des deux dimensions propres aux professions que sont, la nature du savoir et son orientation vers le service attendu par la profession. Déjà en 1963, dans un numéro de la revue de l'Académie des Arts et des Sciences (Daedalus), Barber disait : " Le savoir approfondi et l'orientation vers la communauté qui caractérisent le comportement

\footnotetext{
${ }^{117}$ Cahier des normes pédagogiques nationales du cycle de master. Ministère de l'éducation nationale, de l'enseignement supérieur, de la formation des cadres et de la recherche scientifique (2005). Arrêté n¹810-05 du 10 Chaabane 1426 (15 septembre 2005). Maroc.
} 
professionnel sont devenus indispensables à notre société telle que nous la voyons et la voulons. En vérité, notre type de société ne peut aujourd'hui conserver son caractère propre qu'en élargissant le champ des comportements professionnels." ${ }^{118}$

Le caractère professionnel du métier d'enseignant est depuis quelques décennies mis en exergue dans la formation des enseignants (B.Wentzel 2009, P. Perrenoud 2001). En conséquence, le développement de la dimension pratique de la formation est devenue l'objet d'une réflexion didactique de la profession.

$\mathrm{Au}$ Maroc, comme dans plusieurs autres pays, le profil de l'enseignant est défini à travers un certain nombre de compétences établies par des spécialistes de l'éducation puis institutionnalisé à travers un dispositif national de qualification. La mise en œuvre de ce dispositif se fait au sein des Centres Régionaux des Métiers de l'Éducation et de la Formation (CRMEF).

Etant donnée la diversité des définitions de la compétence, nous avons retenu la définition de la compétence exprimée ainsi par Marguerite Altet (2006): "L'ensemble des ressources cognitives, affectives, motrices mobilisées pour faire face à une famille de situations-problèmes ».

L'enseignant dans son activité professionnelle est censé disposer d'un certain nombre de ressources cognitives, affectives et motrices qu'il devra mobiliser pour faire face à des familles de situations-problèmes. Pour cela, les études en cycle de Master d'enseignement offrent à l'étudiant la possibilité de s'approprier un ensemble de ressources (des savoirs, des savoir-faire et attitudes) en rapport avec la discipline de spécialité. Il a aussi l'opportunité d'acquérir des connaissances en didactique de la discipline et des savoirs théoriques en sciences de l'éducation. C'est la mobilisation de toutes ces ressources en situation professionnelle qui fera l'objet de notre travail. Nous souhaitons, à travers le stage, amener l'étudiant à réfléchir sur la mise en oeuvre de toutes ces ressources dans un contexte professionnel. Notre principal objectif est donc de l'aider à développer des compétences professionnelles liées au métier d'enseignant telles que décrites par la littérature internationale ${ }^{119}$.

Un enseignant agit avec professionnalité lorsqu'il sait faire face à des situations professionnelles diverses (d'enseignement, de gestion pédagogique et/ou administrative, de communication avec les différents partenaires...).

Il agira avec professionnalisme s'il est muni des outils nécessaires pour rester professionnel. Il devra, entre autres, savoir mener son enseignement en maîtrisant les aspects didactiques, relationnels et

\footnotetext{
118 . Bourdoncle R, 1993.

${ }^{119}$ La formation à l'enseignement professionnel, Les orientations, les compétences professionnelles. Gouvernement du Québec, Ministère de l’Éducation (2001), 2001 - 0100649, ISBN : 2-550-38260-9, Dépôt légal - Bibliothèque nationale du Québec.
} 
gestionnaires de la vie de classe, maîtriser la composante juridique et légale qui gère sa fonction, considérer la composante éthique liée à l'exercice du métier, savoir prendre les bonnes décisions en toutes circonstances, tenir compte des différences et des similitudes des partenaires (acteurs et usagers du système éducatif). Le professionnalisme est intimement lié à l'enseignant bien plus qu'à un cadre systémique, pédagogique et juridique. L'enseignant devrait être imprégné de la philosophie et l'essence de sa profession.

Les formations universitaires de certains corps de métier (médecins, ingénieurs, avocats...) intègrent des périodes d'apprentissages supervisées. En milieu hospitalier, le jeune médecin est rarement seul à prendre en charge les cas les plus difficiles.

En revanche, dès la fin de sa formation, le jeune enseignant est confronté à une situation d'enseignement similaire à celle de ses collègues les plus expérimentés. Parfois même, il récupère les classes les plus difficiles et enseigne dans des conditions bien plus délicates que celles de ses collègues bénéficiant du prestige de leur « ancienneté » dans le métier ${ }^{120}$.

Dans ce sens, le travail de préparation à la pratique du métier via le stage proposé se veut un moyen d'atténuer la profondeur du fossé qui sépare la formation de l'enseignant et l'exercice de sa fonction.

Pour cela, l'identification des compétences jugées importantes pour l'acquisition de ressources requises et leur mobilisation dans l'exercice de la fonction d'enseignant est la première étape du processus menant à la professionnalisation. Par suite, la structuration des compétences identifiées en un référentiel de compétences semble être un outil intéressant pour le suivi du développement de celles-ci chez l'étudiant.

Notre principal objectif étant de mener le stagiaire à développer une pensée réflexive sur ses actes professionnels. Pour l'amener vers une conscientisation de ses responsabilités futures, la mise en place d'un dispositif de formation s'avère utile.

\section{Constats et difficultés relevés lors de l'expérience antérieure}

Lors de la formation des enseignants telle qu'elle se déroulait au sein de notre établissement (l'école normale supérieure), le suivi du stage se résumait à une interprétation pédagogique des gestes à avoir aussi bien lors des exercices pratiques réalisés au sein de notre institution (cours de didactique et micro enseignement) que lors des séances d'accompagnement et de discussion qui suivent l'acte d'observation et/ou l'acte de prise en main de la classe en milieu scolaire. L'attente du retour de ce stage se concrétisait sous la forme d'un document écrit nommé "rapport de stage".

${ }^{120}$ Huberman M., 1993. 
En vue de dépasser la réduction du stage et surtout de son rapport tel qu'il se faisait au seul aspect descriptif et restitutif, nous avons tenu à intégrer une composante réflexive à cette activité. Pour cela, le contexte international de professionnalisation du métier d'enseignant a orienté nos choix. Le modèle de formation retenu est basé sur un accompagnement menant au développement de compétences en rapport avec le métier. La composante réflexive du stage est essentielle pour la validation de ces compétences. C'est pour cela et pour s'inscrire dans un contexte socio-constructiviste que nous parlons de développement et non seulement d'acquisition de compétences. Nous souhaitons que le stagiaire puisse développer des stratégies pour acquérir et améliorer ces compétences professionnelles tout au long de sa vie active.

Notre expérience dans le domaine de la formation d'enseignants nous a permis de constater qu'un travail important et consistant se faisait lors de la partie pratique de la formation. Néanmoins, cet investissement ne se reflétait pas à sa juste valeur dans les documents élaborés par les étudiants pour rendre compte de leur expérience. La valorisation de ces efforts consentis par les deux parties, enseignants et stagiaires, restait limitée. Dans le but d'optimiser le travail et de canaliser les énergies des enseignants et des stagiaires, nous avons élaboré un dispositif de formation intégrant un document d'accompagnement dans lequel nous avons explicité chacune des compétences du référentiel.

\section{Expérience de la certification C2i}

Conscient de l'importance qu'occupe le numérique dans le monde actuel, le système éducatif français a mis en place un dispositif certifiant de la capacité minimale à user de l'outil chez ses étudiants, en l'occurrence le $\mathrm{C} 2 \mathrm{i}^{121}$ (Certificat Informatique et Internet).

Notre implication directe en tant que membres actifs du comité de mise en place de la certification C2i "niveau1" et "niveau2 enseignant" au sein de l'université Cadi Ayyad nous a amenées vers une conscientisation effective du rôle que peut jouer un référentiel de compétences dans leur développement. En effet, grâce à cette expérience, nous avons pu constater à quel point la structuration des attentes d'une exigence sous forme de compétences permettait l'appropriation des ressources et leur mobilisation dans des situations d'action. Parfois, l'individu détient cette compétence mais il n'en a pas toujours conscience.

Le processus de certification C2i niveau2 enseignant (C2i2e) a été le principal moteur qui nous a conduites à élaborer des outils de cadrage de

\footnotetext{
${ }^{121}$ http://www.c2i.education.fr/spip.php?rubrique1
} 
l'expérience pratique (stage) menée par chaque étudiant ayant suivi la formation en Master d'enseignement.

La réflexion menée par Marguerite Altet ${ }^{122}$ à propos de la place du C2i2e dans la formation des enseignants au sein des IUFM en France est venue assoir notre pressenti à propos de la nécessité de définir des attentes claires et précises du déroulement, de l'accompagnement et de l'évaluation du stage. Selon M. Altet (2006), « Assurer une formation professionnalisante c'est aider à construire un répertoire de savoirs professionnels spécifiques au métier, intégrant savoirs issus de l'expérience pratique et savoirs scientifiques, pédagogiques et didactiques pour permettre le développement des compétences en contexte d'action. »

\section{Dispositif de formation}

La formation dans les filières universitaires d'éducation (FUE) telle qu'elle a été conçue par l'ENS de Marrakech favorise l'opérationnalisation de ce processus par une approche théorique abordée au sein des modules de didactique et des sciences de l'éducation mais aussi par une approche pratique (stage) dans laquelle l'étudiant a l'occasion de mobiliser les ressources acquises en contexte d'action.

En vue de pallier aux insuffisances relevées dans le mode de fonctionnement antérieur, nous avons mis en place un dispositif qui initie l'étudiant aux diverses composantes du métier d'enseignant et dont l'axe central est un référentiel de compétences.

\section{Référentiel de compétences pour le stage}

Notre ambition n'est point de créer de toutes pièces un référentiel de compétences mais plutôt de s'inspirer de l'existant dans les divers domaines pour l'adapter à notre situation particulière, à savoir la préparation au métier d'enseignant du secondaire qualifiant (élèves 15-18 ans) du Maroc. Nous tenons à préciser que le stage a pour ambition d'approcher la compétence en tant que processus générateur du produit fini qu'est la performance ${ }^{123}$.

Le référentiel se décline en deux grands domaines de compétences. Le premier apporte une réflexion sur l'environnement dans lequel évoluera le futur enseignant. Le second concerne des compétences liées à l'aspect pratique du métier d'enseignant.

\section{Domaine A : "compétences liées au contexte du métier"}

Il se décline en trois compétences qui visent la sensibilisation du stagiaire aux principales composantes de son environnement de travail d'un

\footnotetext{
${ }^{122}$ Altet M, 2006

${ }^{123}$ Wittorski R, 2002.
} 
point de vue administratif, relationnel et communicationnel, éthique et juridique.

- $\quad$ La compétence relative à l'aspect administratif vise l'identification des fonctions et rôles des divers intervenants de son environnement professionnel ainsi que la connaissance des lieux et leurs usages à l'échelle locale, régionale et nationale.

- $\quad$ Une autre compétence concerne une sensibilisation aux divers modes de communication et de relations à entretenir avec les acteurs et les usagers du système éducatif.

- La troisième compétence concerne une initiation aux règles et responsabilités éthiques et juridiques du professionnel de l'éducation envers les différents acteurs et usagers du système éducatif.

\section{Domaine B : "Compétences relatives à la pratique de l'enseignement"}

Il se décline en cinq compétences aidant à développer un esprit à la fois opérationnel et critique de pratiques d'enseignement. Les compétences sont elles-mêmes détaillées en items. . À terme, l'objectif est de développer chez le stagiaire :

- Un esprit critique via des situations d'observation de classe doublée d'une réflexion argumentée faisant référence à des productions scientifiques reconnues;

- $\quad$ la capacité de concevoir et préparer des contenus d'enseignement en identifiant des situations d'apprentissage adaptées au public et à l'avancement dans le programme;

- $\quad$ la capacité de mise en œuvre et gestion pédagogique de contenus. Le stagiaire aura à mener des situations d'apprentissage diversifiées en gérant les temps et les modalités de travail en prenant en compte la diversité des élèves.

- $\quad$ La capacité de prévoir et mettre en œuvre une démarche d'évaluation diversifiée. Il s'agit de lui faire prendre conscience progressivement des différents types d'évaluation à mettre en œuvre tout au long de son enseignement.

- La capacité de travailler en collaboration et de tenir compte de l'aspect évolutif du métier d'enseignant. Le stagiaire est invité à se mettre en condition de manière individuelle et collective pour favoriser un développement professionnel et se tenir au courant des avancées pédagogiques et scientifiques de son domaine et de sa discipline.

Par ailleurs et toujours à travers cette dernière compétence, il s'agit de développer chez le futur enseignant l'esprit de collaboration-encadrement en vue de soutenir les nouveaux arrivants dans le même esprit que le médecinprofesseur encadre le médecin nouvellement diplômé. Le professeur 
d'accueil en acceptant de jouer ce rôle enclenche ce processus. Il faudra par l'intermédiaire de cette formation amener l'étudiant à intégrer cette dimension de son métier pour accompagner d'autres nouveaux arrivants dans le champ de l'éducation.

\section{Document d'accompagnement}

L'élaboration d'un référentiel de compétences suppose la mise en place d'un document d'accompagnement qui viendra expliciter les attentes du référentiel pour aboutir à la validation des compétences et de leurs items. Cet outil aura le mérite de permettre le rapprochement des visions des évaluateurs pour une meilleure homogénéité de l'évaluation.

\section{Rencontres réflexives en amont et en aval}

De quelle manière ce suivi pourrait-il être opérationnalisé de façon structurée pour atteindre les objectifs escomptés? Des rencontres hebdomadaires sont organisées en présentiel pour réfléchir ensemble sur les situations de mise en pratique. Dans l'idée de permettre une implication optimale des stagiaires dans la découverte de l'exercice de la profession, le stage a été monté de telle sorte que la partie en présentiel soit l'occasion de travailler en amont les expériences à vivre et d'analyser en aval les expériences vécues.

\section{Activités de validation}

La validation des compétences du référentiel passe par la réalisation de travaux visant plusieurs items de compétences. Chacun des sept travaux proposés se compose de cinq rubriques :

- $\quad$ Présentation

- Objectifs

- $\quad$ Compétences visées

- Travail à réaliser

- Documents à produire

Tous ces travaux devront être remis sur la plateforme dans un portfolio individuel.

\section{Modalités de validation}

La validation des compétences se base sur une évaluation de type formative qui permet à l'étudiant de mener un processus réflexif de développement des compétences.Pour opérationnaliser cette façon d'agir, les travaux sont remis tout au long du semestre avec une possibilité de retour formatif. Le dernier travail se veut être une synthèse de tous les aspects de l'expérience vécue avec un retour réflexif. Certaines compétences sont visées et validées grâce à l'accomplissement d'un seul travail et d'autres à caractère 
transversal le sont à travers plusieurs travaux. La validation choisie est de type acquis/non acquis.

\section{Plateforme de suivi à distance et d'évaluation}

Le suivi de l'activité du stage se déroulent à travers l'usage d'une plateforme d'enseignement à distance afin de s'affranchir des contraintes du présentiel. Dans notre expérimentation, les volets de l'usage de la plateforme que nous avons privilégiés sont le suivi et l'évaluation. D'autres expériences ont montré que l'usage d'une plateforme permettait le "renforcement de la motivation et de la confiance de l'acteur dans le dispositif de formation du stage $^{124}$.

Une première plus-value de la plateforme réside dans le fait qu'elle impose la définition préalable d'un ensemble de critères et d'éléments observables en termes de savoirs, savoir-être et savoir-faire. L'évaluation consiste alors en la confrontation de ces critères avec les éléments observables dans les productions des stagiaires. Notre choix est conforté par l'expérience menée dans le cadre du développement d'une plateforme de suivi et d'évaluation tout en s'appuyant sur le fait que "l'évaluation d'une compétence (Paquay, 2002 ; Tardif, 2006) consiste aussi à émettre un jugement de valeur parmi différents choix possibles sur la base de la comparaison d'un faisceau de traces, d'indices, d'éléments ou d'aspects observables (valeurs d'indicateurs prédéfinis) avec un référent (ensemble de critères prédéfinis)."125

Une seconde plus-value réside dans le fait que nous avons voulu que l'évaluation soit formative avec un aspect de régulation en vue de validation.

\section{Mise en œuvre du plan de formation}

Le processus de validation du référentiel de compétences s'inscrit dans un plan de formation dédié au stage. Ce plan édicte le processus de formation et les règles d'évaluation. Dans ce processus, une partie se déroulera en présentiel et une autre en distanciel.

Dans le cadre du stage, les étudiants et l'équipe d'encadrement (coordonnateur du stage, accompagnateur-encadrant, professeur d'accueil) ont chacun un rôle à jouer pour aboutir à l'accomplissement du processus. Nous entendons par :

- Coordonnateur : l'enseignant de l'ENS qui supervise administrativement et pédagogiquement l'ensemble des activités du stage - $\quad$ Accompagnateur-encadrant : l'enseignant de l'ENS qui accompagne et encadre pédagogiquement les activités du stagiaire

\footnotetext{
${ }^{124}$ Jullien J-M., Martel C. , Heutte J. \& Alin C., 2010.

${ }^{125}$ Jullien J.M, Martel C, Heutte J, Alin C, 2010.
} 
- $\quad$ Professeur d'accueil : l'enseignant du lycée qui reçoit et accompagne le stagiaire dans ses activités au sein de l'établissement d'accueil.

- $\quad$ Stagiaire : étudiant en filière universitaire d'éducation.

Nous estimons que l'équipe d'encadrement doit aider le stagiaire dans sa réflexion sur son enseignement mais aussi dans la "construction de routines, de procédures" ${ }^{126}$. Les savoir-faire et savoir-être identifiés par les professionnels de l'éducation seront appréciés chez le stagiaire par les formateurs impliqués dans cette activité. Le contexte de formation met en place des "dispositifs d'analyse de pratiques" ${ }^{127}$ via des rencontres entre l'accompagnateur-encadrant, le professeur d'accueil et le groupe de stagiaires.

Le stage se veut être l'occasion de revenir en pratique sur des concepts théoriques abordés lors de la formation de l'étudiant.

Le référentiel de compétences est déposé sur la plateforme d'elearning puis explicité en présentiel. Nous avons jugé utile de préciser les attentes du stage à travers le document d'accompagnement du référentiel et les activités de validation. Parallèlement, les étudiants sont amenés à concevoir et mettre en œuvre des séquences d'enseignement qu'ils exposeront en présence de leurs collègues et de l'équipe d'encadrement au sein de l'institution de formation. S'en suivent des séances de discussion sur la séquence observée. A travers cette expérience, le stagiaire est initié à la conception, mise en œuvre, observation et analyse critique d'une séance d'enseignement.

En alternance avec ces séances en présentiel, les stagiaires observent puis interviennent dans diverses institutions d'accueil (lycées ou institutions de l'enseignement qualifiant, élèves 15-18 ans) sous l'encadrement du formateur "accompagnateur-encadrant" et du "professeur d'accueil". Ces travaux sont évalués via la plateforme. L'enseignant coordonnateur du stage y dépose les textes des travaux à réaliser, les stagiaires peuvent y consulter le référentiel de compétences et doivent y remettre leurs travaux. S'ensuit alors un aller-retour formatif entre le stagiaire et son enseignant évaluateur pouvant aboutir à la validation des compétences.

\section{Retour sur l'expérience (analyse et résultats)}

Ce dispositif a fait l'objet d'une expérimentation avec les étudiants de deuxième année du cycle de master en enseignement des mathématiques à l'école normale supérieure de Marrakech.

Dans ce premier travail, nous nous contentons de rapporter les impressions des étudiants sur le stage tel qu'il s'est déroulé. Nous réunirons

\footnotetext{
${ }^{126}$ Altet M, 2006

${ }^{127}$ Altet M, 2006
} 
les principales idées exprimées dans les rapports finaux du stage élaborés par ces derniers. Nous les présenterons en deux catégories : avantages et difficultés.

\section{Avantages}

Les stagiaires affirment que le stage a été pour eux l'occasion de :

- $\quad$ prendre contact avec l'environnement de travail et ses diverses composantes

- $\quad$ s'immerger dans le milieu de l'enseignement

- découvrir les moyens de communication avec les divers partenaires du système éducatif

- établir des relations de collaboration avec les intervenants pédagogiques déjà en place

- $\quad$ réaliser que le travail collaboratif permet de mieux appréhender diverses situations

- $\quad$ prendre conscience de l'obligation d'agir en respect des règles d'éthiques liées à la profession

- $\quad$ renforcer la confiance en soi

Les impressions recueillies ci-dessus témoignent du fait que le processus d'acquisition des compétences relevant du domaine A semble enclenché.

Par ailleurs, les étudiants affirment que le stage a été pour eux l'occasion de :

- $\quad$ tirer profit de l'expérience de l'équipe d'encadrement,

- $\quad$ prendre conscience de la composante affective de l'enseignement,

- $\quad$ prendre la responsabilité pour concevoir et mettre en œuvre des contenus d'enseignement,

- $\quad$ acquérir des compétences en termes de préparation et présentation de séquences d'enseignement,

- $\quad$ user des logiciels dédiés à l'enseignement des mathématiques (Géogebra) pour faciliter l'appréhension de certains concepts

- $\quad$ prendre la parole en présence d'élèves,

- $\quad$ prendre conscience des différentes composantes de la gestion de classe: prise de parole organisée, interactivité, mise en confiance de l'élève...,

- $\quad$ prendre conscience que l'enseignement ne se limite pas à la seule maitrise de la discipline,

- $\quad$ Se rendre compte de la difficulté que rencontre l'enseignant pour motiver les élèves,

- Découvrir la manière d'agir face aux erreurs,

- $\quad$ être critique vis-à-vis des pratiques d'enseignement, 
- $\quad$ se maintenir au courant des avancées scientifiques concernant la discipline et sa didactique en usant des techniques de l'information et de communication (TIC),

- $\quad$ prendre conscience de ses forces et faiblesses sur le plan personnel et scientifique,

- $\quad$ user des TIC pour interagir avec les formateurs via la plateforme.

Les impressions recueillies dans le deuxième groupe ci-dessus témoignent du fait que le processus d'acquisition des compétences relevant du domaine $B$ semble enclenché.

Une dernière impression recueillie chez l'un des stagiaires résume l'idée qu'il se fait de "l'enseignant qui réussit dans l'exercice de son métier" : "un enseignant qui réussit dans l'exercice de son métier est un enseignant qui a les compétences dans les domaines suivants : compétences liées à la planification et la préparation de leçons, compétences liées à la mise en œuvre de séance d'enseignement, compétences liées à la mise en place de bonnes relations humaines de l'enseignant avec ses élèves et des élèves entre eux, compétences en termes de remise en question et d'auto critique".

\section{Difficultés} suivants

A la fin de leur stage, les étudiants ont relevé les points faibles

- $\quad$ Insuffisance du nombre de séances de prise en main de la classe;

- Les interventions ayant eu lieu exclusivement dans le système éducatif privé ${ }^{128}$, ont donné une vision tronquée du système éducatif marocain;

- $\quad$ L'architecture du master prévoit d'autres modules d'enseignement en parallèle du stage. Les stagiaires estiment que cela a pour effet de réduire leur temps d'immersion dans le milieu éducatif.

Ce premier retour de l'expérience montre que les étudiants ont d'ores et déjà pressenti la dimension de professionnalité et professionnalisme du métier. En effet, certaines de leurs impressions donnent des indicateurs qui pourraient en témoigner. On note aussi l'émergence d'une forme de professionnalité et de professionnalisme dans leur comportement. Les stagiaires semblent prendre conscience de diverses facettes du métier : relationnelles, communicationnelles, collaborationnelles, gestionnelles, ... Dans leur analyse, ils perçoivent qu'il est nécessaire de disposer d'un savoir approfondi mais estiment que sa seule maîtrise n'est pas suffisante. Ils prennent également conscience que l'orientation vers la communauté pour laquelle ce savoir est destiné est prépondérant.

${ }^{128}$ Absence de convention institutionnelle permettant l'accès aux établissements de l'enseignement public. 


\section{Conclusion}

Cette expérience nous a permis de faire divers constats.

On note une réelle prise de conscience de diverses composantes de l'acte d'enseignement.

La précision des attentes du stage à travers le dispositif élaboré cadre et oriente à la fois le travail de l'équipe d'encadrement et celui des stagiaires.

A travers les activités de validation rendues, nous relevons l'émergence d'une réflexion intéressante sur le métier d'enseignant.

Nous notons que le suivi du stage est facilité dans une grande proportion par l'usage d'une plateforme d'enseignement à distance.

L'évaluation formative a été un facteur déterminant dans le développement de la pensée réflexive sur le métier d'enseignant.

Néanmoins, l'infrastructure et la planification du stage dans le cursus de formation ont posé quelques difficultés ayant pu entraver l'optimisation de la mise en œuvre du dispositif.

Nous espérons pouvoir généraliser ce processus à l'ensemble des filières accréditées au sein de notre établissement et améliorer la gestion pédagogique de la composante professionnelle de la formation à l'enseignement.

Un objectif ultérieur serait d'étendre le processus enclenché dans cette pré-expérimentation en vue de développer un outil de formation continue tout au long de la vie.

\section{References:}

Cahier des normes pédagogiques nationales du cycle de master. Ministère de l'éducation nationale, de l'enseignement supérieur, de la formation des cadres et de la recherche scientifique. Maroc, Arrêté n¹810-05 du 10 Chaabane 1426 (15 septembre 2005).

Dispositif de qualification des enseignants au sein des Centres Régionaux des Métiers de l'Éducation et de la Formation (CRMEF) au Maroc, document cadre.

La formation à l'enseignement professionnel, Les orientations, les compétences professionnelles. Gouvernement du Québec, Ministère de l'Éducation, 2001 - 01-00649, ISBN : 2-550-38260-9, Dépôt légal Bibliothèque nationale du Québec, (2001).

Jullien J.M, Martel C, Heutte J, Alin C, 2010 Dispositif de certification de compétences dans l'enseignement supérieur : interrogations de la pedagogie universitaire induites par le programme recherche et développement EmaEval. Actes du congrès de l'Actualité de la recherche en éducation et en formation (AREF), Université de Genève, septembre 2010. 
Formation des enseignants : Référentiel de compétences professionnelles. Direction de la formation. Haute école pédagogique du canton de Vaud. (2011).

Référentiel national du certificat informatique et internet de l'enseignement supérieur de niveau 2 «enseignant». Bulletin officiel n5, France, (2011).

Dispositif de formation des professeurs fonctionnaires stagiaires. Rectorat de Reims, Portfoli_tuteur. Reims, (2011).

Altet M. La place de l'évaluation des compétences dans la formation des enseignants : Le C2i2e. Séminaire C2i2e Nantes, (2006).

Altet M., Guibert P. \& Perrenoud P., Formation et professionnalisation des métiers de l'éducation et de la formation. Revue recherche en éducation, n'8. (2010).

Altet M, Séminaire C2i2e - NANTES - 13 et 14 novembre 2006

Bourdoncle R. La professionnalisation des enseignants : les limites d'un mythe. Revue Française de Pédagogie, ${ }^{\circ}$ 105, octobre-novembre-décembre (1993)

El Gharras S et Charki J, (2014) Dispositif de formation et d'évaluation du stage de fin de formation, Filière universitaire d'éducation, Niveau master d'enseignement. Actes du 26ème colloque de l'ADMEE Europe, janvier 2014, Marrakech.

Huberman M. Enseignement et professionnalisme: des liens toujours aussi fragiles. Revue des sciences de l'éducation, vol. XIX, no 1, 1993, p. 77 à 85. Canada, (1993).

Jullien J-M., Martel C. , Heutte J. \& Alin C. Dispositif de certification de compétences dans l'enseignement supérieur : interrogations de la pédagogie universitaire induites par le programme recherche et développement Emaeval. $26^{e}$ congrès international d'actualité de la recherche en éducation et en formation (AREF), Genève (Suisse), (2010).

Martinet M-A, Raymond D. \& Gauthier C, La formation à l'enseignement : Les orientations. Les compétences professionnelles. Ministère de l'éducation. Gouvernement du Québec, (2001).

Perrenoud, $\mathrm{Ph}$, Des savoirs aux compétences : de quoi parle-t-on en parlant de compétences ? Pédagogie collégiale (Québec), Vol. 9, n 1, octobre, pp. 20-24, (1995).

Perrenoud Ph, Construire un référentiel de compétences pour guider une formation professionnelle, Faculté de psychologie et des sciences de l'éducation, Université de Genève, (2001).

Perrenoud $\mathrm{Ph}$. Évaluer les compétences en situation de travail : aussi nécessaire que difficile! Genève, (2006).

Wentzel B. Référentiels de compétences et modèles de professionnalités pour l'enseignement secondaire. Revue-Formation et pratiques d'enseignement en questions. No 15 / 2012 / pp. 135-158. Suisse, (2012). 
Wittorski R, La professionnalisation. Revue-Savoirs, 2008/2. n 17, p. 9-36, (2008).

Wittorski R, De la fabrication des compétences, (2002).

\section{Sitographie}

http://www.c2i.education.fr/

http://www.iufm.fr/reseau-iufm/tice/actes/2006-

nantes/documents/altet/index.swf

http://ressources.lachiver.fr/c2i2e/presentation/web/co/competence.html

http://www.recherches-en-education.net/spip.php?article121

https://plone2.unige.ch/aref2010/communications-orales/premiers-auteursen-j/Dispositif\%20de\%20certification.pdf

hal.archives-ouvertes.fr/docs/00/17/26/96/DOC/art-edpte-135.DOC

http://www.cairn.info/revue-savoirs-2008-2-page-9.htm

www.hepl.ch 